\title{
Asteroid and NEA detection models
}

\author{
R. Michelsen ${ }^{1}$, H. Haack ${ }^{2}$, A. C. Andersen ${ }^{3}$, and J. L. Jørgensen ${ }^{4}$ \\ ${ }^{1}$ Astronomical Observatory, NBIfAFG, Copenhagen University, Juliane Maries Vej 30, 2100 Copenhagen Denmark, \\ E-mail: rene@astro.ku.dk \\ ${ }^{2}$ Geological Museum, Copenhagen University, Øster Voldgade 5-7, 1350 Copenhagen K, Denmark, E-mail: \\ hh@savik.geomus.ku.dk \\ ${ }^{3}$ NORDITA, Blegdamsvej 17, 2100 Copenhagen, Denmark, E-mail: anja@nordita.dk \\ ${ }^{4} Ø$ rsted*DTU,MIS, Building 327, Technical University of Denmark, 2800 Lyngby, Denmark, E-mail: jlj@ oersted.dtu.dk
}

\begin{abstract}
We determine the possible detection rate of asteroids with the Bering mission. In particular we examine the outcome of the Bering mission in relation to the populations of NearEarth Asteroids and main belt asteroids. This is done by constructing synthetic populations of asteroids, based on the current best estimates of the asteroid size-distributions. From the detailed information obtained from the simulations, the scientific feasibility of Bering is demonstrated and the key technical requirement for the scientific instruments on Bering is determined.
\end{abstract}

\section{Introduction}

The Bering mission is an autonomous mission, with the purpose of making sample observations of the inner asteroid populations. In particular, Bering will travel through most of the space between Venus and the outer parts of the asteroid belt at 3.5 AU [9], and will thus be able to observe members of the Near-Earth Asteroid (NEA) population, objects in the asteroid main belt, and objects en route from the main belt toward the NEAs. The instruments on board the spacecrafts are the Advanced Stellar Compass (ASC) [12] that allows Bering to detect and follow moving objects, with the purpose of orbit determination of the objects. In addition, the ASC can control a small telescope [13], so that observations of the objects can be obtained. In this way, Bering is able to provide both an orbit as well as a physical characterisation of the objects. The key point is the autonomy of the ASC and the telescope, that enables Bering to systematically detect and follow objects down to object diameters at the meter level [12, 13]. The scientific objectives have been discussed in detail elsewhere [1].

One of the key issues to address is the two-fold question of how many objects Bering will be able to observe. First, this question is important to answer as a basis for the scientific objectives, and with the need to describe the scientific feasibility. Second, the number of objects detected will depend on the limiting magnitude of the ASC, and is thus vital for the technical requirements to the mission, also in terms of the number of objects that needs to be processed by the autonomous spacecraft platform. It is however not trivial to answer this question [1], hence we have a constructed a simulator capable of examining the detailed aspects of the detections.

There already exists a number of simulations of the detection of NEAs [16, 11], however these simulations differs from our needs in several ways. First of all, they are made for ground based surveys for NEA discovery and followup. For Bering, we need to be able to simulate observations made by a spacecraft in an interplanetary orbit. Also, the simulations are normally strongly restricted in the size of the objects included. For the Bering mission, we need to have detailed knowledge of for how long time an object can be observed, as well as the angular velocity. For instance, the trailing losses experienced by ground based surveys due to fast moving objects across the field of view during the exposure, are addressed by the Bering ASC capabilities to handle fast moving objects, however we need to quantify the requirements to the ASC.

Thus, we in general need to understand how Bering will perform when inserted into a given asteroid population, and with the possibility of adjusting parameters, like the limiting detection magnitude and the orbit. We shall here focus on synthetic populations of the NEAs and the main belt asteroids. The synthetic objects are treated as massless test particles in the simulation, and after an initial sorting, the objects are numerically integrated. This allows a careful examination of the requirements to the ASC when probing members of these populations down to the meter-range.

\section{Asteroid magnitudes and diameters}

As discussed [1], one of the challenges when observing asteroids are their rapid and drastic variations in magnitude. The magnitude of an asteroid does not only depend on physical parameters like the size and the albedo, but also on the distances to the Sun and the observer, and the phase angle. Depending on the geometry, there can thus be a strong time dependency on the magnitude variations.

The magnitudes of asteroids are normally described by a two-parameter model. The absolute magnitude $H$ of an asteroid is defined as the $\mathrm{V}$-magnitude at unit distance to the Sun $(r)$ and the Earth $(\Delta)$, and at phase angle $\alpha=0$. The 
second parameter is the slope parameter $G$, which is an expression for the geometric albedo. Thus the relation between the observed V-magnitude and $H$ and $G$ is [4]

$$
\begin{aligned}
V= & H+5 \log r \Delta \\
& -2.5 \log \left[(1-G) \Phi_{1}(\alpha)+G \Phi_{2}(\alpha)\right]
\end{aligned}
$$

where $\Phi_{1}$ and $\Phi_{2}$ are functions of the phase angle $\alpha . r$ and $\Delta$ are measured in AU.

Only for a few objects has the slope parameter $G$ and the albedo $p_{V}$ been measured. The desire however persists to translate the absolute magnitude $H$ into a diameter $D$ of the object. This can be done by an a priori assumption on the albedo of the object. The diameter can then be expressed as [5, 20]

$$
D(\mathrm{~km})=1329 \cdot 10^{-H / 5} / \sqrt{p_{V}}
$$

where $D$ is in units of $\mathrm{km}$. Care is needed when evaluating the sizes of the smallest sub-kilometer asteroids, as the actual albedo of these objects is currently not known.

\section{The NEA population}

During recent years, many publications have been made in the attempt of establishing the size distribution of NearEarth Asteroids. Bottke and collaborators [3] have constructed a de-biased estimate of the NEA size distribution, for the range up to absolute magnitude $H=22$, by simulating a ground based NEA survey, and taking into account the nature of the source regions of the NEAs. For this range, they find a best fit of the form $\log N=\alpha H+\beta$, where $N$ is the accumulated number of objects and $\alpha$ and $\beta$ are constants. Werner [20] provides an estimate for the distribution up to $H=30(D=3 \mathrm{~m})$, derived from the lunar crater sizefrequency distribution. Their distribution corresponds well to the estimates obtained from ground based NEO surveys [18]. Werner obtains a distribution similar to that of Bottke, however at around $H=21$ the distribution shows a turning point, with a much steeper slope. For the even smaller end, Brown and collaborators [5] reports satellite observations of bolide detonations in the Earth's atmosphere, for objects in the range of $1 \mathrm{~m}$ to $100 \mathrm{~m}$. The distribution derived from these observations is consistent with the work of Werner.

It should be stressed that the estimated populations are based on indirect methods, and that various factors may influence the actual, present size distribution. One of the factors is the translation from $H$ to $D$, as the albedo $p_{V}$ of the NEA population is more or less unknown. There are indications, for instance in the work of Werner, that the smallest NEAs have larger albedo. This is compatible with our hypothesis that the smallest asteroids are young collisional fragments without surface dust, and with minimum exposure to cosmic radiation, hence the objects have larger albedo.

The distribution we have adopted for the NEA population is a combination of the estimation by Bottke, combined with the work by Werner, supported by the measurements by Brown. We shall thus split the distribution into three parts,

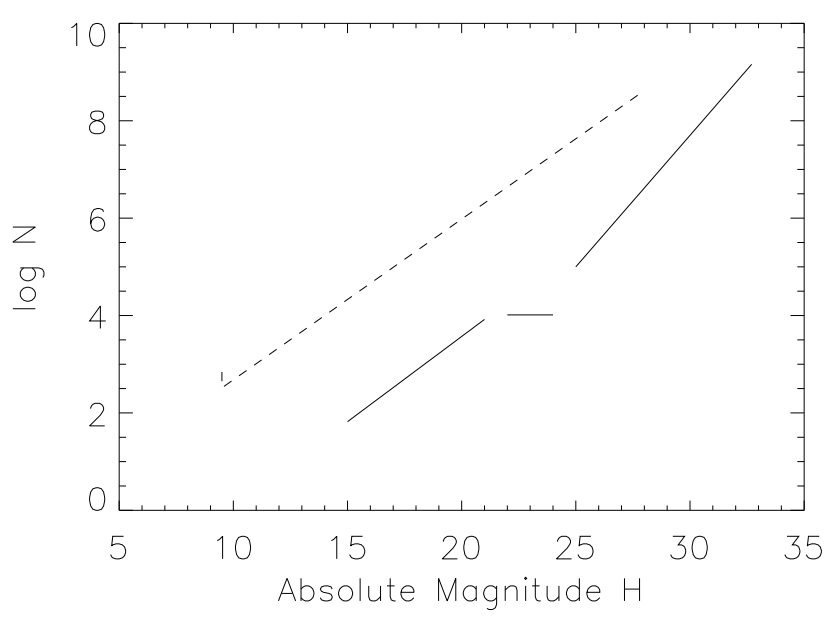

Figure 1. The absolute magnitude $(H)$ distribution of the asteroid populations of our simulations. $N$ is the accumulated number of objects. The solid line is the NEA population, the dashed line is the main belt distribution. For the NEA population, we adopt a three-step distribution, based on the indications of a steeper increase in the number of the smaller objects, compared to the larger objects. The main belt follows a simple linear distribution in $(H, \log N)$, however with certain limitations of this distribution (see text for discussion).

with bins of 1 magnitude, as

$$
\begin{aligned}
\log N & =0.35 H-3.43 & & ; 15 \geq H<22 \\
N & =10303 & & ; H=[22,24] \\
\log N & =0.54 H-8.5 & & ; 24<H \leq 32.7
\end{aligned}
$$

The distribution can be seen in Fig. 11 The first part is thus adopted from Bottke, followed by a plateau. The last part of the distribution is taken from the low-albedo distribution of Werner. The $H=32.7$ has been chosen, so that with an albedo of $p_{V}=0.17$, this corresponds to a diameter of $D=1 \mathrm{~m}$. The total number of objects in the distribution amounts to $\log N=9.158$, or approximately $1.4 \times 10^{9}$ objects. For the slope parameter we initially assumed $G=0.15$, a low-albedo population, but later we made tests with a high-albedo population using $G=0.32$, corresponding to $p_{V} \approx 0.25$ (see the discussion of the results below).

Besides the distribution in terms of $H$, the de-biased distribution by Bottke also suggests a spatial distribution of the objects, in terms of the eccentricity, the semi-major axis and the inclination. However, for the above synthetic population, we have selected a uniform distribution of the orbital elements (within the definitions of a Near-Earth Object). In principle, this is a failure of the distribution, relative to the current best estimates. However, the suggested distribution of the orbital elements is only valid for objects with $H<22$. There is only vanishing statistical material for smaller objects (larger $H$ ), hence there is no basis to assume a continuation of the distribution in the orbital elements for $H>22$. In any case, the number of objects with $H \leq 22$ amounts to approx. 18600 , a vanishing number compared to the total number of objects. 


\section{The main belt population}

The main belt population is very much different from that of the NEAs. First of all, our observational knowledge is strongly restricted to only the very largest objects, due to the far distances to the objects.

Durda and collaborators [8] have made numerical models of the collisional evolution of the main belt asteroids. The observed distribution shows two bumps relative to a linear $(D, \log N)$ fit, for objects with $D=100 \mathrm{~km}$ and $D=5 \mathrm{~km}$. By adjusting the parameters of their models, Durda is able to reproduce these bumps, and thus to obtain an estimate of the main belt distribution down to $1 \mathrm{~m}$ in size. Other estimates for the distribution exists (see [7] for an overview), and they tend to agree for objects down to $1 \mathrm{~km}$.

For these simulations we shall adopt the distribution by Durda, with some modifications. The focus of our simulations are on the sub-kilometer objects, hence for the range $H=[5 ; 10]$ we count the total number of known objects [10], and assign to them the absolute magnitude $H=9.5$. This involves 694 of the largest known objects, and causes an initial bump in the distribution (Fig. 11). We then make a linear fit to the distribution of Durda for their size range $100 \mathrm{~m}$ to $50 \mathrm{~km}$,

$$
\log N=0.33 H-0.62
$$

and apply this distribution for the magnitude interval $H=$ [10;27.94] in steps of 0.5 magnitudes. Using the albedo referred to by Durda of $p_{V}=0.1178$, the $H=27.94$ corresponds to an object size of $D=10 \mathrm{~m}$. However, for the slope parameter we use $G=0.15\left(p_{V}=0.17\right)$, a slightly larger albedo than used by Durda, so that the smallest object in our distribution in fact has $D=8 \mathrm{~m}$.

This distribution has two flaws. First of all, it does not take the mentioned known bumps of the large objects into account. However, the number of objects among the large sizes neglected in this manner is completely negligible in comparison with the total number of objects in this synthetic population. The large objects are as well not the main objective of this work, but should under all circumstances be treated separately, taking into account the already known objects. The works by Durda and by Davis [7] seems to indicate an increase in the slope of the size distribution for $D<100 \mathrm{~m}$. Whether this holds true or not is one of the scientific objectives of Bering. However, it means that relative to these estimates, our synthetic main belt population is heavily underestimating the number of $10 \mathrm{~m}$ objects, with a factor of 10 or more. This must be taken into account, when reviewing the results of the simulations.

The total number of objects in the main belt population is thus $\log N=8.6$, or around $4 \times 10^{8}$ objects. The orbital elements are assumed to be uniformly distributed.

\section{The numerical approach}

With the large number of objects involved in estimating the detection rate, a direct numerical integration is not feasible due to the very heavy demands on CPU time and disk space. Instead, we adopted a solution that provide a rough sorting

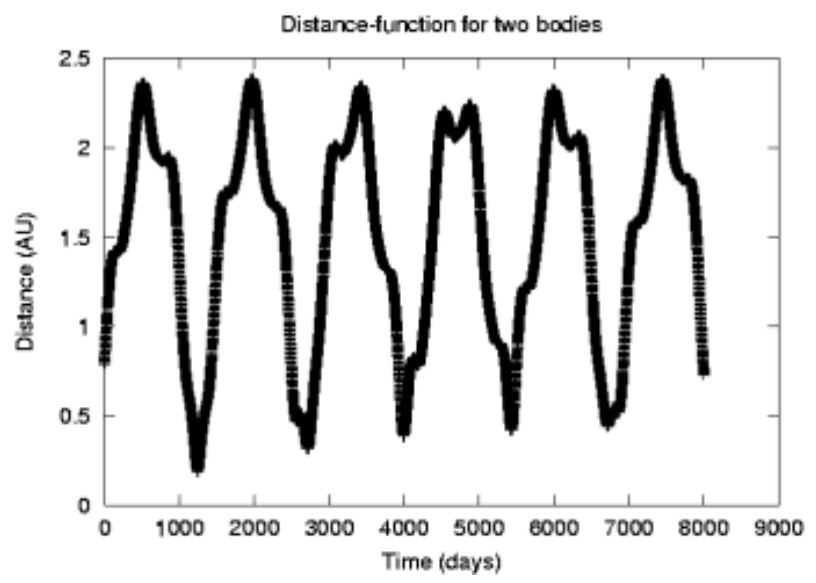

Figure 2. Example of the distance variation between Bering and a synthetic object (a test particle). The MOID is the global minimum of the distance.

of the objects, discarding those that would not become observable from Bering within a reasonable time. A simple idea is to calculate the minimum orbital intersection distance (MOID) between the Bering orbit and every object. If the MOID distance is sufficiently small, so that in a best case situation the object is observable within the magnitude limits, the object is selected for subsequent detailed analysis, otherwise it is discarded. Instead of a purely numerical approach for determining the MOID, for instance by integrating the orbits and calculating the smallest distance between Bering and the objects, we use a semi-analytic approach, implementing the MOID method suggested by Sitarsky [19]. For two Keplarian orbits, the distance function can be written as

$$
f(V, v)=\frac{1}{2}(\mathbf{R}-\mathbf{r})^{2},
$$

where $(V, v)$ are the true anomalies of the objects of concern, and $\mathbf{R}, \mathbf{r}$ are the cartesian coordinates relative to some origin. By examining the derivatives of this function, Sitarsky derives analytical equations for the minimum of the function, the MOID. These equations can, for the general case, not be solved analytically, hence we apply a version of the Newton-Raphson method that is suited for non-linear cases (Press et al. [17]), and in particular ensures convergence toward some minimum. This problem is however not trivial. Often, the solution must be sought in very rough terrain, Fig. 2 hence one set of initial conditions may not be enough to ensure that the global minimum is found. Thus we solve the equations for several sets of initial conditions, and from the resulting local minima, we accept the smallest value as the MOID. We verified the implementation of the MOID method against a number of different orbit scenarios, and compared with the minimum distance between the two orbits, obtained by direct numerical integration.

After the MOID has been calculated, we apply a selection criteria for the sorting. We calculate the $V$-magnitude of the asteroid by using the MOID as the distance to the observer. The perihelion distance, the smallest distance to the Sun, is used as the asteroid-Sun distance. Further, the phase angle is set to zero. This is truly a best case configuration of the asteroid. If the calculated magnitude is $V \leq V_{\lim }$, where $V_{\text {lim }}$ is the Bering detection limit, the object is saved, otherwise 
it is discarded.

This MOID-sorting is done for all the objects. It is computationally heavy, but allows focus on only the most interesting objects. As the sorting can be quite aggressive, depending on $V_{\text {lim }}$, it can drastically decrease the demands for computing time and disk space for a detailed analysis of the Bering detection rate.

The objects that were selected by the MOID sorting for further analysis, are endured to a numerical integration. For each time step, the V-magnitude of the objects is calculated, and compared against $V_{\text {lim }}$, disregarding the direction to the object, thus assuming that Bering is able to monitor the whole sky. This is a good approximation, as the only directions Bering will not cover are toward the Sun, in which direction only the night side of the objects will be seen, thus being too faint under all circumstances. During the integration, book keeping is made of which objects had $V<V_{\text {lim }}$, the smallest $V$ of the objects and the corresponding tangential velocity during the minimum $V$. By running a small filter after the integration, a complete list of the observable objects could be generated from the book keeping data.

As integrator, we used the SWIFT package [14, 15]. The package was modified to allow handling of the magnitude calculations and of the book keeping data. As the primary integrator we used the symplectic RMVS3 method, however for selected objects we made a refined check with the classic Bulirsch-Stoer method [17], without any noticeable difference in the results of the two integrators.

Due to the very transient variations in brightness of the smallest objects, the integrations were typically made over 50 days, with a data dump and magnitude calculation every 0.02 days (28.8 minutes).

The MOID implementation and the numerical integrations were parallelized at the run-level, and ran on the IBM RS6000 cluster of the Center for Scientific Computing Aarhus (CSC-AA) [6].

\section{Results for the NEAs}

The first key problem to deal with was to assess the value of $V_{\lim }$. This value is critical for the mission feasibility in terms of the the detection rate of the Bering Advanced Stellar Compass, i.e. the number of objects that would be observed per unit of time. If $V_{\lim }$ is too small, only a small number of objects would be found during the mission life time, and the science telescope would remain idle most of the time. If $V_{\text {lim }}$ is too large, it would exhaust the onboard system, for instance requiring faster CPUs with larger power consumption, violating the power budget.

Due to the heavy computations involved of the full asteroid populations, we decided to initially work on a small subset of the NEAs, by extrapolating the Bottke distribution, Eq. $3 \mathrm{a}$, to an object size of around $1 \mathrm{~m}, H \approx 32.5$, for a total of exactly $10^{8}$ objects. Many of the ground based surveys are able to reach $V=19$, so this value we used as the initial $V_{\text {lim }}$ in the MOID and the integration. As the orbit of Bering, we simply used the Earth orbit, for comparison with the ground based surveys. The results are shown as Run 1 in Table 1 Of the initial 100 million objects, only 7 million, or $7 \%$, remains after the MOID sorting. Of these objects, 619 were seen by Bering over 50 days. The detection rate would thus be 12.4 objects/day. For each object we know the circumstances of the detection, for instance the smallest object observed had $H=32.66, D=1 \mathrm{~m}$, and the object had $V<V_{\text {lim }}$ for 144 minutes. At the brightest point $V=18.5$, and the object was moving with $25.2 \% / \mathrm{sec}$. For each of the 619 objects we thus know the maximum brightness of the objects during the 50 days, and we can then determine the threshold of $V$ where the detection rate goes to zero. In this manner, we find that for $V_{\text {lim }}=15,17$ objects could be observed, corresponding to a detection rate of 0.3 objects/day. Taking into account that this run is a factor of 14 short in the number of objects, we would expect a detection rate of approximately 1 object/day using the full population Eq. 3 with $V_{\lim }=15$.

Run 2 utilizes the full NEA population Eq. 3 with $V_{\lim }=$ 15. From the original approx. $1.4 \times 10^{9}$ objects, around 10 million, or $0.7 \%$, were left after the MOID sorting. We find that 16 objects could be seen over 50 days, amounting to a detection rate of 0.3 objects/day. This number is the same as for the test population, Run 1. However for Run 1 three objects have $D \leq 10 \mathrm{~m}\left(H=27.68, p_{V}=0.17\right)$ whereas of the 16 objects in Run 2, 8 have $D \leq 10 \mathrm{~m}$, and 3 have $D=1 \mathrm{~m}$.

These results we can compare to the observations by Brown and collaborators [5]. According to their work, approx. 30 objects/yr collides with the Earth, with diameters $D \geq 1 \mathrm{~m}$. To "collide with the Earth" would in the case of Bering mean that the object is detected $\left(V \leq V_{\text {lim }}\right)$ and encounters Bering within a distance equal to the Earth radius. On the other hand, an object within that distance is not necessarily seen by Bering, as the magnitude of the object depends on the phase angle and the distance to the Sun and Bering (an object does not need to have $V<V_{\text {lim }}$ in order to collide with the Earth). Hence, in the best case, for an object with $D=1 \mathrm{~m}$ at $1 \mathrm{AU}$ distance to the Sun and at $\alpha=0$, the detection radius of Bering would be $43260 \mathrm{~km}$ at $V=15$ and $6856 \mathrm{~km}$ at $V=11$. For $V=11$, we find three objects in that range, at $V=11.42, V=11.70, V=11.74$ during the 50 days time span. This amounts to 22 objects/year, comparable to the 30 objects/yr by Brown et al. If we strictly look at the diameter, for $D \leq 4 \mathrm{~m}$, the Earth is hit one time per year according to Brown et al. Hence the Earth is hit by 29 objects/year with $1 \mathrm{~m} \leq D \leq 4 \mathrm{~m}$. In that size range, we have from the simulation that $6 \mathrm{obj} / 50 \mathrm{~d}$ were detected, equal to $44 \mathrm{obj} / \mathrm{yr}$. With the differences between "colliding with the Earth" and "observing an object", and the short integration time span in mind (Brown et al. is based on $8.5 \mathrm{yr}$ of observations), we are quite convinced that our simulations are consistent with the observations.

For objects smaller than $1 \mathrm{~m}$ it has been suggested [2] that the Earth is hit by one object per day with $D \approx 15 \mathrm{~cm}$. For Bering, such an object would have $V \leq 15$ within a distance of $6548 \mathrm{~km}$.

An even more detailed integration of the smallest object of Run 2 reveals that the object would be visible for $43 \mathrm{~min}$ utes, with a close approach distance of $19500 \mathrm{~km}$. The angular velocities of the smallest of the objects were in the range 100-300"/sec. In comparison, a $15 \mathrm{~cm}$ object would be moving with $619 \% / \mathrm{sec}$ at the maximum detection distance. 
Table 1. Results of the simulations for the NEA and main belt populations. $N$ is the initial number of objects, $N_{\text {MOID }}$ is the number of objects remaining after the MOID sorting and $N_{\mathrm{obs}}$ is the number of objects that could be seen during the integration time for the stated values of $V_{\lim }$ and $G . t$ is the time span and $t_{\mathrm{dump}}$ is the time resolution of the integration. For Run 3, the object population was adopted from Run 2, however with a change in $G$. The population of Run 5 is the same as for Run 4, however with different parameters for the numerical integration.

\begin{tabular}{|l|c|c|c||c|c|}
\hline \multirow{2}{*}{} & \multicolumn{3}{|c|}{ NEA population } & \multicolumn{2}{c|}{ Main belt population } \\
\cline { 2 - 6 } & Run 1 & Run 2 & Run 3 & Run 4 & Run 5 \\
\hline$V_{\lim }$ & 19.0 & 15.0 & 15.0 & 15.0 & 15.0 \\
$\mathrm{G}$ & 0.15 & 0.15 & 0.32 & 0.15 & 0.15 \\
$t$ (days) & 50 & 50 & 50 & 356 & 50 \\
$t_{\text {dump }}($ days) & 0.02 & 0.02 & 0.02 & 1.0 & 0.02 \\
$N$ & $10^{8}$ & $\approx 1.4 \times 10^{9}$ & - & $\approx 4 \times 10^{8}$ & - \\
$N_{\text {MOID }}$ & $\approx 7 \times 10^{6}$ & $\approx 10^{7}$ & - & $\approx 10^{7}$ & - \\
$N_{\text {obs }}$ & 619 & 16 & 23 & 647 & 352 \\
\hline
\end{tabular}

From these considerations, we find that a detection limit of $V_{\lim }=15$ would provide high feasibility of the Bering mission. Concerning the large angular velocities, there may however be a conflict between the detection limit and the fastest moving objects, due to trailing losses. The capabilities of the ASC to handle fast moving objects at the suggested limiting detection magnitude remains to be studied.

As it was mentioned previously, there are strong indications that the albedo of the smallest asteroids is relatively large. To check the influence of the albedo on the detection rate, we for Run 3 simply changed the slope parameter of the MOID-sorted objects from Run 2, for a direct comparison. $G$, the slope parameter, was changed from 0.15 to 0.32 , corresponding to a change in albedo from approx. 0.17 to 0.25 . In this run, 23 objects were detected, 7 more than in Run 2 , corresponding to a detection rate of 0.46 objects/day. 14 objects were smaller than $H>28\left(D<7 \mathrm{~m}, p_{V}=0.25\right)$. In Run 2, 8 objects had $H>28$, hence 6 of the 7 additional objects belongs to the small end of the size range. This indicates that the albedo may influence the detection rate by a factor of two. This, on the other hand, may mean that the detection rate measured by the Bering mission will not only provide a constraint on the size/absolute magnitude distribution, but also on the albedo. Particularly in the case where the Bering observations are consistent with the ground based observations, the albedo can be determined directly from the count statistics. This question should be address by a more detailed analysis, involving integrations over longer time spans.

\section{Results for the main belt}

With the value of $V_{\lim }=15$ found suitable for the NEA population, we continued to examine the capabilities of the mission with such a limiting magnitude in the asteroid main belt. Initially, Bering was kept at a circular orbit in the ecliptic, with a semi-major axis of $2.5 \mathrm{AU}$. This places Bering in the inner part of the main belt.

A first run, Run 4, was made to test the behavior of the simulations. Of the 400 million objects, 11 million or $2.7 \%$ survived the MOID. The integration was made over 356 days, with a resolution of 1 day. 647 objects were seen, i.e. a detection rate of $1.8 \mathrm{object} / \mathrm{day}$. Of these, 204 were smaller than $10 \mathrm{~km}(H>12.94)$, on the other hand, none of the smallest objects were seen, the smallest having $H=27.00$ $\left(D=15 \mathrm{~m}, p_{V}=0.17\right)$. Hence only a fraction of the largest objects could be seen over one year. It remains to be examined how many of the largest objects that can be observed during the mission life time.

The smallest objects were presumably not observable due to the transient brightness behavior upon a close encounter, and were thus not found as the time between each magnitude calculation $\left(t_{\text {dump }}\right)$ is too large. Hence we made a second run, Run 5 , with a time span of 50 days and with a resolution of 0.02 days. This time, 352 objects could be seen, or 7 objects/day. Of these, 55 have $H>12.94$, so at least on object smaller than $10 \mathrm{~km}$ could be seen per day. The smallest object found had $H=27.00$, and was visible for 6 hours, so none of the $H=27.94$ objects could be seen. As mentioned, this may be due to the underrepresentation in the number of these objects, in combination with the short integration time span. It should be examined whether any of the $H=27.94$ objects can be seen by extending the integration time span. The tangential velocities of the encountered objects were typically in the range of 5-20 "/sec.

\section{Conclusion}

We have analyzed the scientific feasibility of the Bering mission, when applied to the Near-Earth Asteroids and the asteroid main belt. We have found that with a limiting magnitude of the ASC of approx. $V_{\lim }=15$, the scientific feasibility can be sustained. This would allow the detection of around one object per day, in both the NEA and main belt populations. This initial detection by the ASC will allow the science telescope to be pointed toward the object, and detailed observations can be initiated, both in terms of physical characterisation, but also in terms of following the object toward faint magnitudes.

For the main belt population, we find that there is room for lowering $V_{\text {lim }}$ if e.g. required by the smaller power budget.

Some issues however still remains open, in particular the capabilities of the ASC to follow very fast moving objects at faint magnitudes. It also remains to be examined how Bering will perform when placed in the proposed eccentric orbit [9] ranging from $0.7 \mathrm{AU}$ at perihelion to $3.5 \mathrm{AU}$ at aphelion. In addition to the already outlined scientific objectives [1], we also find that the albedo of the object 
populations has an influence on the detection rate, and the question of whether the albedo can be derived directly from detections among the NEA population needs a further close analysis, as this is critical for the physical characterisation, including the object size.

\section{Acknowledgments}

Computing facilities were provided by the Aarhus branch of the Danish Center for Scientific Computing (CSC-AA). This work was supported by the Danish Natural Science Research Council through a grant from the Center for GroundBased Observational Astronomy (IJAF).

\section{References}

[1] Andersen A.C., Michelsen R., Haack H., Jørgensen J.L., 2003, "Bering - The first deep space mission to map asteroidal diversity, origin and transportation", these proceedings.

[2] Bland P.A., Artemleva N.A., 2003, Nature 424, 288291

[3] Bottke W.F.Jr., Jedicke R., Morbidelli A. et al., 2000, Science 288, 2190

[4] Bowell E., Hapke B., Domingue D., Lumme K., Peltoniemi J., Harris A.W., in Binzel R.P., Gehrels T., Matthews M.S., Asteroids II, 1989, The University of Arizona Press

[5] Brown P., Spalding R.E., ReVelle D.O., Tagliaferri E., Worden S.P., 2002, Nature 420, 294-296

[6] Center for Scientific Computing Aarhus, http://www.cscaa.dk/

[7] Davis D.R., Durda D.D., Marzari F., Campo Bagatin A., Gil-hutton R., 2002, in W.F. Bottke, A. Cellino, P. Paolicchi, R.P. Binzel (eds.) Asteroids III, The University of Arizona Press

[8] Durda D.D., Greenberg R., Jedicke R., 1998, Icarus $135,431-440$

[9] Hansen F., Thomsen P.L., 2003, "The Bering mission trade-off and scale", these proceedings

[10] Jedicke R., Larsen J., Spahr T., 2002, in W.F. Bottke, A. Cellino, P. Paolicchi, R.P. Binzel (eds.) Asteroids III, The University of Arizona Press

[11] Jedicke R., Morbidelli A., Spahr T., Petit J.-M., Bottke W.F.Jr., 2003, Icarus 161, 17-33

[12] Jørgensen J.L., Denver T., Betto M., Jørgensen P.S., 2003, "The Bering autonomous target detection", these proceedings

[13] Jørgensen J.L., Denver T., Betto M., Jørgensen P.S., 2003, "The Bering target tracing instrumentation", these proceedings

[14] Levison H., 2003, http://www.boulder.swri.edu/ hal/swift.html

[15] Levison H.F., Duncan, M.J., 1994, Icarus 108, 18

[16] Muinonen K., 1998, Planet. Space. Sci. 46, 291-297
[17] Press W.H., Teukolsky S.A., Vetterling W.T., Flannery B.P., 1992, Numerical Recipes, 2nd ed., Cambridge University Press

[18] Rabinowitz D., Helin E., Lawrence K., Pravdo S., 2000, Nature 403, 165-166

[19] Sitarsky G., 1968, Acta Astron. 18, 171-205

[20] Werner S.C., Harris A.W., Neukum G., 2002, Icarus 156, 287-290 